\title{
Investigation of Carrier Transport in GaN Single Crystals and Radiation Detectors by Thermally Stimulated Methods
}

\begin{abstract}
V. Kažukauskas*, V. Kalendra and J.-V. Vaitkus
Semiconductor Physics Department

and Institute of Materials Science and Applied Research

Vilnius University; Saulètekio al. 9, bldg. 3, 2040 Vilnius, Lithuania

We investigated single crystals of $\mathrm{GaN}$ and thin film GaN radiation detectors by thermally stimulated currents and thermally stimulated depolarization methods in order to characterize carrier transport properties as influenced by material defect structure. In thick GaN no expressed structure of the thermally stimulated current spectra was observed in the temperature range from $100 \mathrm{~K}$ up to $350 \mathrm{~K}$, which could be characteristic of the thermal carrier generation from trap levels. The experimental facts imply that the thermally stimulated current spectra might be caused not by carrier generation, but it could be due to thermal mobility changes. Therefore we had applied the numerical analysis by taking into account carrier scattering by ionized impurities and phonons. It was found that mobility limited by ionized impurities varies as $\sim T^{2.8}$ and lattice scattering causes the dependence $\sim T^{-3.5}$. The highest mobility values were up to $1550 \mathrm{~cm}^{2} /(\mathrm{V} \mathrm{s})$ at 148-153 K. Such high values indicate relatively good quality of the single GaN thick crystals. In high resistivity GaN detectors irradiated by high doses of high-energy neutrons and X-rays current instabilities were observed which could be caused by the change of carrier drift paths in a highly disordered matter. A model of carrier percolation transport is presented.
\end{abstract}

PACS numbers: 72.20.Jv, 72.20.Fr, 72.80.Ey, 78.70.--g, 85.30.De

\section{Introduction}

Over the past decade, GaN and related nitrides have been in the focus of intense research [1-3]. Being direct, large bandgap materials, they lend themselves to a variety of electronic and optoelectronic applications. The advantages associated with a large bandgap include higher breakdown voltages, lower noise generation, and high temperature operation. Therefore GaN is one of the most

*corresponding author; e-mail: vaidotas.kazukauskas@ff.vu.lt 
promising materials for high-temperature, high frequency, and radiation hard applications. GaN-based ultraviolet visible-blind photodetectors, blue, violet, and green light emitting diodes, lasers, diodes, and transistors of different types have been demonstrated. Nitrides are ideal for applications in displays and high density data storage.

On the other hand, compared with their technological applications, fundamental research in nitrides, particularly in electronic transport, partly due to poor material quality, appears to be far behind. Technologically defect density is still very high. Spontaneous polarization is present in these wurtzite semiconductors and thus large built in electrostatic fields are induced in layered nitride structures, even in the absence of any strain. Therefore, apart from technological advances in material production, further progress in the development, design and optimization of GaN-based devices necessarily requires a detailed investigation of transport and defect properties by different methods. So, it was our task to investigate single GaN crystals and thin film GaN radiation detectors in order to characterize carrier transport properties as influenced by material defect structure.

\section{Samples and experiment}

We investigated single GaN crystals and thin semi-insulating (SI) GaN structures by means of thermally stimulated currents (TSC), thermally stimulated depolarization (TSD), and current-voltage $(I-V)$ techniques. Single high resistivity GaN platelets with thickness of up to $\sim 500 \mu \mathrm{m}$ were from Lumilog (France). Their carrier concentration ranged from $5 \times 10^{14} \mathrm{~cm}^{-3}$ up to $5.5 \times 10^{15} \mathrm{~cm}^{-3}$ and mobility was $380-920 \mathrm{~cm}^{2} /(\mathrm{V} \mathrm{s})$. Thin epitaxial SI-GaN detector structures were grown by MOCVD technology on a sapphire substrate. They consisted of a $2.5 \mu \mathrm{m}$ SI-GaN upper layer on a $n$-GaN buffer. Gold Schottky contacts were evaporated on the top. They were irradiated with $100 \mathrm{keV}$ reactor neutrons $\left(5 \times 10^{14} \mathrm{~cm}^{-2}\right)$ and $10 \mathrm{keV}$ X-rays (600 Mrad).

For the thermally stimulated measurements the samples were cooled down to liquid nitrogen temperature. Later on they were excited either by a white light or applied voltage. After the excitation and the relaxation in the dark, the samples were heated at a constant rate of $10 \mathrm{~K} / \mathrm{min}$. To reveal the possible influence of the overlapping maxima that could be caused by several competitive processes, we applied the fractional heating technique. This advanced modification is a powerful tool for the discrimination of the overlapping thermally stimulated processes in materials with many levels in the bandgap (e.g., [4]). The multiple heating enables the sequential emptying of the initially filled shallower levels thus giving the information about the deeper ones in the repetitive temperature scans. The polarization effects were investigated by TSD.

\section{Results and discussion}

TSC curves of the single crystal GaN platelets are presented in Fig. 1. It is noticeable that no expressed structure of the TSC spectra was observed in 


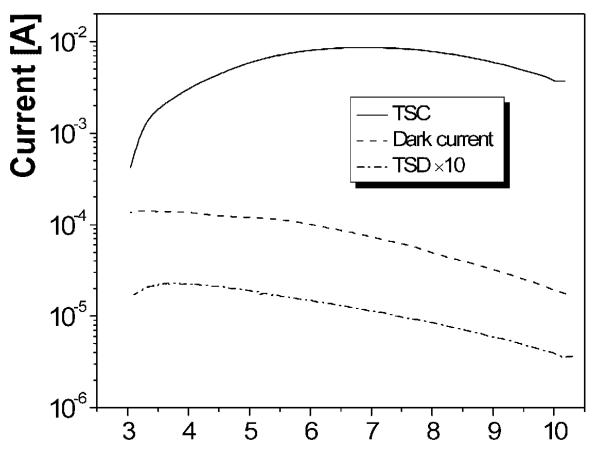

Fig. 1

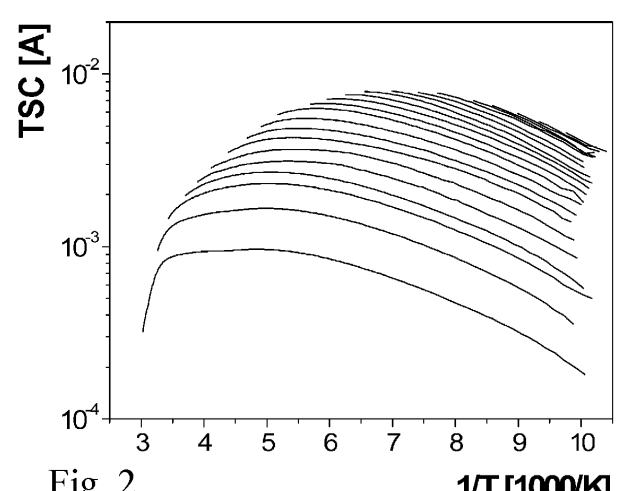

Fig. 1. Thermally stimulated, dark current and TSD spectra in a single GaN crystal. For the sake of convenience TSD current is multiplied by a factor of 10 .

Fig. 2. Thermally stimulated current spectra obtained by repetitive heating of the single GaN crystal.

the temperature range from $100 \mathrm{~K}$ up to $350 \mathrm{~K}$ that could be characteristic of the thermal carrier generation from trap levels. Instead, only one wide and flat maximum used to appear. Moreover, TSCs in $\log I \sim 1 / T$ scaling, where $I$ is the current and $T$ is the temperature, were not linear, in contrast to the classical thermal generation model from trap levels $[4,5]$. In order to reveal the possible structure of this maximum we applied the fractional heating technique that is a powerful tool for the discrimination of overlapping maxima [4]. Nevertheless by repetitive thermal scans the shape of the TSC maximum did not change, as it is demonstrated in Fig. 2. Furthermore, application of the TSD method resulted in a similar dependence of the depolarization current as it is shown in Fig. 1.

These facts imply that most probably the measured dependences were caused not by carrier thermal generation, but rather by their mobility variation. A similar conclusion that illumination even at $77 \mathrm{~K}$ has a very little effect on the electron density in GaN but can lead to a noticeable persistent increase of the Hall mobility was made in Ref. [6]. The induced persistent photoconductivity effect was, therefore, attributed to the Hall mobility through intrinsic electrically active defects. The authors also report the very strong ionized impurity scattering that limits the Hall mobility in GaN. Very similar mobility variation with temperature were obtained in Ref. [7]. In this paper authors fit experimental mobility data presented in Ref. [8], by taking into account the ionized impurity and phonon scattering.

The first step of the modeling consists in an approximation of the doping level dependence of the mobility [7] on the base of the known Caughey-Thomas approximation [9]:

$$
\mu_{i}(N)=\mu_{\min , i}+\frac{\mu_{\max , i}-\mu_{\min , i}}{1+\left(N / N_{g, i}\right)^{\gamma_{i}}}
$$

where $i=n, p$ for electrons and holes, respectively. Model parameters $\mu_{\max , i}$, 
$\mu_{\min , i}, N_{g, i}$, and $\gamma_{i}$ depend on the type of semiconductor material, and $N$ is the doping concentration.

On the other hand, at low doping level and room temperature phonon scattering is prevailing. In this case $\mu_{\max , i}=\mu_{I, i}$. The temperature dependences of mobilities limited respectively by phonons and ionized impurities were supposed to follow the known rules [10]: $\mu_{\mathrm{L}}=\mu_{\mathrm{L}}\left(T_{0}\right)\left(T / T_{0}\right)^{-\alpha}$ and $\mu_{\mathrm{I}}=\mu_{\mathrm{I}}\left(T_{0}\right)\left(T / T_{0}\right)^{-\beta}$. Here coefficient $\alpha$ describes the lattice scattering effect, meanwhile $\beta$ characterizes the ionized impurity scattering. Usually in real semiconductors the values of $\alpha$ and $\beta$ are not equal to that evaluated theoretically, and therefore they have to be found experimentally.

The following expression was derived to model mobility, depending on the concentration of scattering centers and temperature [7]:

$$
\mu_{i}(N, T)=\mu_{\max , i}\left(T_{0}\right) \frac{B_{i}(N)\left(T / T_{0}\right)^{\beta_{i}}}{1+B_{i}(N)\left(T / T_{0}\right)^{\alpha_{i}+\beta_{i}}},
$$

where

$$
B_{i}(N)=\left.\left[\frac{\mu_{\min , i}+\mu_{\max , i}\left(N_{g, i} / N\right)^{\gamma_{i}}}{\mu_{\max , i}-\mu_{\min , i}}\right]\right|_{T=T_{0}} .
$$

This model was applied to fit the experimental data, and an excellent agreement was obtained as it is demonstrated in Fig. 3. It was supposed that carrier concentration remains unchanged and equals $1 \times 10^{15} \mathrm{~cm}^{-3}$ as it was indicated by the producer. The fitting parameters are as follows: $\mu_{\max , i}=1600 \mathrm{~cm}^{2} /(\mathrm{V} \mathrm{s})$, $\mu_{\min , i}=160 \mathrm{~cm}^{2} /(\mathrm{V} \mathrm{s}), N_{g, i}=2.7 \times 10^{17} \mathrm{~cm}^{-3}, \gamma_{i}=1.3, a_{i}=3.5, b_{i}=2.85$, $N=1.2 \times 10^{17} \mathrm{~cm}^{-3}, T_{0}=185 \mathrm{~K}$. This means that in our single GaN crystals mobility limited by ionized impurities varies as $\sim T^{2.8}$ and lattice scattering causes the dependence $\sim T^{-3.5}$. The highest mobility values were up to $1550 \mathrm{~cm}^{2} /(\mathrm{V} \mathrm{s})$ at 148-153 K. Such high values indicate a relatively good quality of the crystals. Nevertheless, they were reached only in pre-excited samples. After thermal cycling the experimental maximum used to decrease and to move towards higher
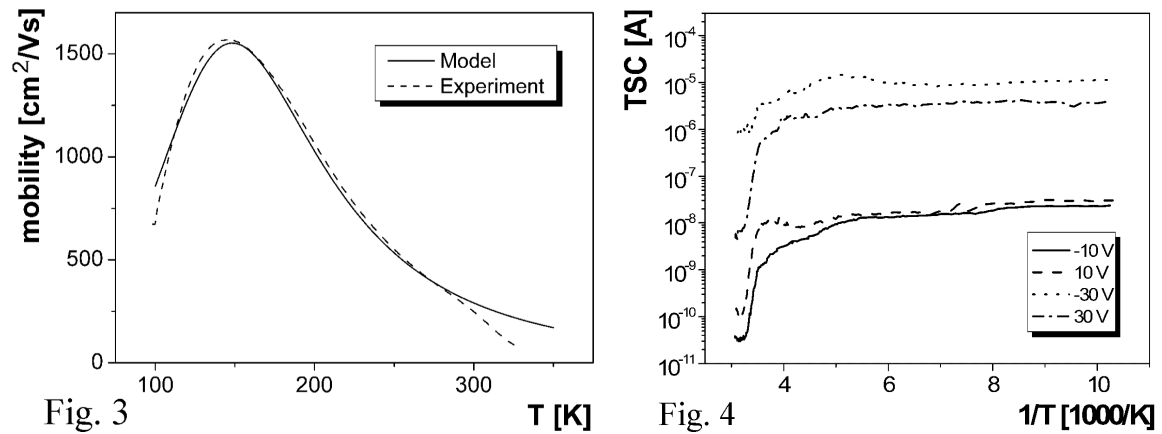

Fig. 3. Numerical fitting (solid curve) of the experimental mobility dependence (dashed curve).

Fig. 4. TSC spectra in GaN detector, irradiated by X-rays. 
temperatures (Fig. 2). Such behavior can be observed if ionized and/or neutral defect scattering is intensifying, e.g., [11]. This can take place in case the effective number of scattering centers was reduced upon excitation. But usually upon excitation the number of ionized impurities, from which carriers were generated, increases. This contradiction is removed if one takes into account the real crystal structure in which not only point defects are present, but more complex defects are formed as well. Usually because of the doping inhomogeneities, which might be due to the minor temperature variation during the growth, defects become distributed inhomogeneously as well. This is a known issue in emerging materials, as it also was, e.g., in semi-insulating GaAs. The inhomogeneity problem in GaAs was solved only after more than one decade of intense investigations. Usually spatial inhomogeneities cause modulation of the bandgap edges and result in the potential relief of the bandgap that plays an effective role in charge transport, e.g., [12-14]. In GaN slow conductivity relaxations were also observed many times that directly evidence the effect of inhomogeneities in transport phenomena. Therefore we propose a model in which the effect of potential fluctuations due to crystal inhomogeneities is involved, causing percolation transport in GaN. At low temperatures carriers generated by light fill potential wells, screening impurities and smoothing the potential relief. Under heating thermally released carriers recombine causing defect inhomogeneities to act as scatterers again. A similar effect of the recapture of excited electrons into illumination-neutralized defects responsible for the persistent mobility in undoped GaN was observed in [6]. The proposed model also explains why nearly no mobility dependence on temperature was observed in GaN samples irradiated by high doses of high-energy neutrons $\left(5 \times 10^{14} \mathrm{~cm}^{-2}\right)$ and by 600 Mrad of X-rays, though their excitation resulted in a current increase up to one order of magnitude (see Fig. 4). This is because of the big number of defects, i.e., inhomogeneities introduced by high-energy irradiation. Furthermore, in these detectors current instabilities were observed that could be caused by the random change of carrier drift paths in a highly disordered material.

\section{Summary and conclusions}

Single crystals of GaN and thin film GaN radiation detectors were investigated by means of the thermally stimulated current and thermally stimulated depolarization techniques in order to identify carrier transport peculiarities resulting from prevailing defects. Experimentally in single high resistivity GaN platelets with thickness of up to $\sim 500 \mu \mathrm{m}$ no expressed structure of the TSC spectra was observed in the temperature range from $100 \mathrm{~K}$ up to $350 \mathrm{~K}$ that could be characteristic of the thermal carrier generation from trap levels. Instead only one wide and flat maximum used to appear. Moreover, TSCs in $\log I \sim 1 / T$ scaling were not linear. In order to reveal the possible structure of this maximum we applied the fractional heating technique as a powerful tool for the discrimination of overlapping maxima. Nevertheless by repetitive thermal scans the shape of the 
TSC maximum did not change. These facts imply that most probably TSCs were caused not by thermal carrier generation, but rather by their mobility variation. Therefore the numerical analysis was applied involving the ionized impurity and phonon scattering. It was found that mobility limited by ionized impurities varies as $\sim T^{2.8}$ and lattice scattering causes the dependence $\sim T^{-3.5}$. The highest mobility values were up to $1550 \mathrm{~cm}^{2} /(\mathrm{V} \mathrm{s})$ at $148-153 \mathrm{~K}$, indicating a relatively good quality of the single GaN thick crystals. Nevertheless they were reached only in pre-excited samples. After thermal cycling the experimental maximum decreased and moved towards higher temperatures. Usually such behavior can be observed if impurity scattering is intensifying. In high resistivity GaN detectors irradiated by high doses of high-energy neutrons $\left(5 \times 10^{14} \mathrm{~cm}^{-2}\right)$ and by $600 \mathrm{Mrad}$ of X-rays nearly no mobility dependence on temperature was observed, though their excitation resulted in a current increase up to one order of magnitude. Furthermore in these samples current instabilities took place that could be caused by the change of carrier drift paths in a highly disordered material. A model of carrier percolation transport is presented, which foresees a significant influence of spatially extended defect complexes and/or associations, leading to the potential inhomogeneities of the bandgap.

\section{References}

[1] S. Nakamura, T. Mukai, M. Senoh, Appl. Phys. Lett. 64, 1687 (1994).

[2] M. Razeghi, A. Rogalski, J. Appl. Phys. 79, 7433 (1996).

[3] I. Akasaki, H. Amano, Jpn. J. Appl. Phys. 36, 5293 (1997).

[4] G. Kavaliauskienè, V. Kažukauskas, V. Rinkevičius, J. Storasta, J.V. Vaitkus, R. Bates, V. O'Shea, K.M. Smith. Appl. Phys. A 69, 415 (1999).

[5] J.G. Simmons, G.W. Taylor, Phys. Rev. B 5, 1619 (1972).

[6] G. Li, S.J. Chua, W. Wang, Solid State Commun. 111, 659 (1999).

[7] T.T. Mnatsakanov, M.E. Levinshtein, L.I. Pomortseva, S.N. Yurkov, G.S. Simin, M. Asif Khan, Solid-State Electron. 47, 111 (2003).

[8] S. Nakamura, T. Mukai, M. Senoh. J. Appl. Phys. 71, 5543 (1992).

[9] D.M. Caughey, R.E. Thomas, Proc IEEE 55, 2192 (1967).

[10] K. Seeger, Semiconductor Physics. An Introduction, 8th ed., Springer, Berlin 2002 .

[11] B.K. Ridley, Quantum Processes in Semiconductors, Clarendon Press, Oxford 1982 .

[12] B. Pistoulet, P. Girard, G. Hamamdjian, J. Appl. Phys. 56, 2268 and 2275 (1984).

[13] V. Kažukauskas, J. Appl. Phys. 84, 2053 (1998).

[14] V. Kažukauskas, J. Storasta, J.-V. Vaitkus, J. Appl. Phys. 80, 2269 (1996). 